\title{
ON THE HÖLDER CONTINUITY OF QUASI- CONFORMAL AND ELLIPTIC MAPPINGS
}

\author{
BY \\ ROBERT FINN AND JAMES SERRIN
}

Let $u(x, y)$ and $v(x, y)$ be continuously differentiable functions defined in a domain of the complex $z$-plane, $z=x+i y$. We shall say that the function $w(z)=u+i v$ represents a quasi-conformal mapping provided there is a positive constant $K<\infty$ such that

$$
u_{x}^{2}+u_{y}^{2}+v_{x}^{2}+v_{y}^{2} \leqq 2 K\left(u_{x} v_{y}-u_{y} v_{x}\right) .
$$

If $K<1$ it is easily seen that $u$ and $v$ are constant; therefore the only values of $K$ that are of interest are $K \geqq 1$. Geometrically, (1) implies that the mapping $z \rightarrow w(z)$ is sense-preserving, and that infinitesimal circles map onto infinitesimal ellipses for which the ratio of minor to major axis is $>K-\left(K^{2}-1\right)^{1 / 2}$. The quantity $\mu=K-\left(K^{2}-1\right)^{1 / 2}$, is thus the greatest lower bound of the "local eccentricity" of all mappings satisfying (1). We shall call $1 / \mu$ the dilatation ratio.

A function $w(z)$ defined in a domain $A$ of the $z$-plane is called Hölder continuous in $A$ with constant $H$ and exponent $\alpha$ if for all pairs of points $z_{1}, z_{2}$, in $A,\left|w\left(z_{1}\right)-w\left(z_{2}\right)\right| \leqq H\left|z_{1}-z_{2}\right| \alpha$. It is known that if $w(z)$ defines a quasiconformal mapping in $A$ then $w(z)$ is Hölder continuous in every compact subdomain of $A$, with exponent depending only on $K$, and with constant depending only on $K$, on a bound for $|w(z)|$, and on distance to the boundary of $A$. Proofs of this fact have been given by Morrey, Lavrentieff, Y ûjôbô, Caccioppoli, Hersch and Pfluger, Nirenberg, Ahlfors, and Mori. (See references at the end of the paper.) In this paper we present another proof of the Hölder continuity of quasi-conformal mappings which has some distinct advantages over the proofs previously given, and which shows, in common with the theory of Hersch and Pfluger, that the Hölder coefficient can be chosen independent of $K$. Precisely, we prove the following result.

THEOREM 1. Let $w=u+i v$ be a quasi-conformal mapping defined in a domain $A$ of the z-plane. Assume that $|w| \leqq 1$. Then in any compact subregion $B$ of $A$, the function w(z) satisfies a uniform Hölder inequality

$$
\left|w\left(z_{1}\right)-w\left(z_{2}\right)\right|<\pi e\left|\frac{z_{1}-z_{2}}{d}\right|^{\mu},
$$

where $\mu=K-\left(K^{2}-1\right)^{1 / 2}, d$ is the distance from $B$ to the boundary of $A$, and e is

Received by the editors November 30, 1956. 
the base of the natural logarithms.

The exponent in this Hölder condition cannot be improved, as is shown by the example $w=r^{\mu} e^{i \theta}$. We emphasize that we do not assume the mapping to be one to one.

We shall treat also a more general class of mappings, namely those which satisfy an inequality of the form

$$
u_{x}^{2}+u_{y}^{2}+v_{x}^{2}+v_{y}^{2} \leqq 2 K\left(u_{x} v_{y}-u_{y} v_{x}\right)+K_{1},
$$

where $K$ and $K_{1}$ are constants, $K \geqq 1$ and $K_{1} \geqq 0$. Mappings which satisfy (2) are important in the theory of elliptic partial differential equations (cf. [7]), and therefore we venture to call them elliptic mappings. In $\$ 1$ we prove the following result concerning the Hölder continuity of elliptic mappings.

TheOREM 2. Let $w=u+i v$ be an elliptic mapping defined in a domain $A$ of the $z$-plane. Assume that $|w| \leqq 1$ and that $K>1$. Then in any compact subregion $B$ of $A$, the function $w(z)$ satisfies a uniform $H$ ölder inequality

$$
\left|w\left(z_{1}\right)-w\left(z_{2}\right)\right| \leqq H\left|z_{1}-z_{2}\right|^{\mu},
$$

where $\mu=K-\left(K^{2}-1\right)^{1 / 2}$, and $H$ depends only on $K, K_{1}$, and the distance from $B$ to the boundary of $A\left({ }^{1}\right)$.

Nirenberg has proved a result similar to Theorem 2, but with a smaller exponent.

It is desirable to have an extension of Theorems 1 and 2 to the case where $w$ is undefined or not differentiable at isolated points of $A$, but otherwise satisfies the same hypotheses. Such a result is proved in $\$ 3$, along with a close analogue of the Riemann theorem on removable singularities of analytic functions.

In the final section of the paper we consider one-to-one quasi-conformal mappings of a domain $A$ onto a domain $B$. It is shown that if $A$ and $B$ have sufficiently smooth boundaries, then any such mapping can be extended so that it is one-to-one and continuous in the closure $\bar{A}$ of $A$; moeover the extended mapping satisfies a uniform Hölder condition over all of $\bar{A}$, with constants depending in a simple way on $A, B$, and $K$ (Theorem 4).

For many purposes it is natural to consider a class of mappings with weakened differentiability requirements. The class $D_{2}$ of Morrey is of particular importance. It is defined by the requirements that (i) $u$ and $v$ be continuous, (ii) $u$ and $v$ be absolutely continuous in $x$ for almost all $y$ and in $y$ for almost all $x$, and (iii) the derivatives $u_{x}, u_{y}, v_{x}, v_{y}$, which exist almost

(1) We have omitted the case $K=1$ from the hypotheses of Theorem 2, because (3) may not hold for this value of $K$ (e.g. $\left.w=(r \log r) e^{i \theta}\right)$. If $K=1$ and $K_{1}=0$ then $w$ is an analytic function of $z$ and, as is known, $\left|w\left(z_{1}\right)-w\left(z_{2}\right)\right|<\left|z_{1}-z_{2}\right| / d$. If $K=1$ and $K_{1}>0$ then for any $\epsilon>0$ the mapping satisfies (2) with $K$ replaced by $K+\epsilon$. Hence an inequality of the form (3) is valid with $\mu=1+\epsilon$. A simple expression for $H$ is given at the end of $\$ 1$. 
everywhere by virtue of (ii), should be square integrable. Condition (1) (or (2)) is then assumed to hold almost everywhere. All results of this paper remain true for these more general mappings. To avoid confusion of ideas we present the proofs under the original assumption of smooth derivatives; in $\S$ A1 of the Appendix we indicate the necessary modification.

1. Proof of Theorem 2. It is convenient to introduce the notation

$$
|\nabla w|^{2}=\left|w_{x}\right|^{2}+\left|w_{y}\right|^{2}=u_{x}^{2}+v_{x}^{2}+u_{y}^{2}+v_{y}^{2} .
$$

We prove first an elementary lemma:

Let $w=u+i v$ satisfy (2) with $K>1, K_{1} \geqq 0$. Then

$$
\left|w_{x}\right|^{2} \leqq \frac{1}{1+\mu^{2}}\left(|\nabla w|^{2}+\frac{2 \mu^{2} K_{1}}{1-\mu^{2}}\right)
$$

where $\mu=K-\left(K^{2}-1\right)^{1 / 2}$.

Proof. Applying Schwarz's inequality to (2), we have

$$
\begin{aligned}
\left|w_{x}\right|^{2}+\left|w_{y}\right|^{2} & \leqq 2 K\left(u_{x} v_{y}-u_{y} v_{x}\right)+K_{1}, \\
& \leqq K\left(\mu\left|w_{x}\right|^{2}+\mu^{-1}\left|w_{y}\right|^{2}\right)+K_{1} .
\end{aligned}
$$

Since $K$ is assumed $>1$, this inequality can be solved for $\left|w_{x}\right|^{2}$; thus $\left({ }^{2}\right)$

$$
\left|w_{x}\right|^{2} \leqq \frac{1}{\mu^{2}}\left|w_{y}\right|^{2}+\frac{2 K_{1}}{1-\mu^{2}},
$$

and it follows that

$$
\left|w_{x}\right|^{2}=\frac{1}{1+\mu^{2}}\left(\left|w_{x}\right|^{2}+\mu^{2}\left|w_{x}\right|^{2}\right) \leqq \frac{1}{1+\mu^{2}}\left(|\nabla w|^{2}+\frac{2 \mu^{2} K_{1}}{1-\mu^{2}}\right) \quad \text { Q.E.D. }
$$

The proof of Theorem 2 is based on an estimate for the growth of the Dirichlet integral

$$
D(r)=\iint_{C_{r}}|\nabla w|^{2} d x d y,
$$

where $C_{r}$ denotes a circle (closed disk) of radius $r$ contained in the domain $A$. In particular, we shall show that $D(r) \leqq$ Const. $r^{2 \mu}$ for $r$ suitably small (cf. inequality (11)); this done, the desired conclusion will follow from a wellknown lemma of Morrey $\left({ }^{3}\right)$. If we integrate (2) over $C_{r}$ there results

$$
D(r) \leqq 2 K \iint_{C_{r}}\left(u_{x} v_{y}-u_{y} v_{x}\right) d x d y+\pi r^{2} K_{1}=2 K \oint u d v+\pi r^{2} K_{1},
$$

(2) In obtaining this and later inequalities it is convenient to use the relation $K=\left(1+\mu^{2}\right) / 2 \mu$.

(3) The lemma in question appears in [6, p. 134]. A statement in a form adapted for this paper, together with a brief proof, are given in $\S A 2$ of the Appendix. 
where the line integral is taken along the circumference $\gamma_{r}$ of $C_{r}$. Let $u_{n}$ be the average value of $u$ on $\gamma_{r}$. Then by Schwarz's inequality

$$
\oint u d v=\oint\left(u-u_{0}\right) d v \leqq \frac{1}{2} \oint\left[\frac{\left(u-u_{0}\right)^{2}}{r}+r v_{s}^{2}\right] d s,
$$

$s$ denoting arc length on $\gamma_{r}$. Also, by Wirtinger's inequality $\left({ }^{4}\right)[4$, Theorem $258 \mid$,

$$
\oint\left(u-u_{0}\right)^{2} d s \leqq r^{2} \oint u_{s}^{2} d s
$$

hence, combining the last three inequalities, we obtain

$$
D(r) \leqq K r \oint\left|w_{s}\right|^{2} d s+\pi r^{2} K_{1} .
$$

Now $x$ in inequality (4) can represent an arbitrary direction, in particular, the direction $s$ in $(6)$; it follows that

$$
D(r) \leqq \frac{r}{2 \mu} \oint|\nabla w|^{2} d s+\pi r^{2} K_{1}\left(1+\frac{2 \mu}{1-\mu^{2}}\right),
$$

or, ecfuivalently

$$
D(r) \leqq \frac{r}{2 \mu} \frac{d D}{d r}+K_{2} r^{2}, \quad K_{2}=\pi K_{1}\left(1+\frac{2 \mu}{1-\mu^{2}}\right) .
$$

Now let $B$ be a closed subregion of $A$, and let $d$ be the distance from $B$ to the boundary of $A$. Consider circles $C_{r}$ whose centers are in $B$; for such circles (7) holds for $0<r<d$. Writing (7) in the form

$$
-\frac{d}{d r}\left(r^{-2 \mu} D\right) \leqq 2 \mu K_{2} r^{1-2 \mu},
$$

and integrating both sides from $r=\rho$ to $r=t$ leads to the inequality

$$
D(\rho) \leqq\left\{D(t)+K_{3}\right\}(\rho / t)^{2}, \quad \rho \leqq t<d,
$$

where $K_{3}=(\mu /(1-\mu)) K_{2} t^{2}$.

An estimate of $D(t)$ can be obtained by returning to the basic formula (5). In virtue of the hypothesis $|w| \leqq 1$, the line integral in (5) has the bound

$$
\begin{aligned}
\oint u d v^{\prime} & =\frac{1}{2} \oint u d v-v d u \leqq \frac{1}{2}\left[\oint|w|^{2} d s\right]^{1 / 2}\left[\oint\left|w_{\bullet}\right|^{2} d s\right]^{1 / 2} \\
& \leqq \frac{1}{2}[2 \pi r]^{1 / 2}[d D / d r]^{1 / 2} .
\end{aligned}
$$

(4) 1 simple proof can be obtained by expanding $\left(u-u_{0}\right)$ in a Fourier series. 
Substituting this estimate into (5), we get $\left({ }^{5}\right)$, for all $r<d$,

$$
\left(D-K_{4}\right)^{2} \leqq 2 \pi K^{2} r \frac{d D}{d r}, \quad K_{4}=K_{1} \pi d^{2} .
$$

Suppose now that $D(t)>K_{4}$; then also $D(r)>K_{4}$ for all $r>t$. We may therefore divide (9) by $\left(D-K_{4}\right)^{2} r$ and integrate both sides of the resulting inequality from $r=t$ to $r=d$. After some simplification there results

$$
D(t) \leqq \frac{2 \pi K^{2}}{\log (d / t)}+K_{4},
$$

and this bound also holds if $D(t) \leqq K_{4}$. We now fix $t=d e^{-\nu}$, where $\nu=1 / 2 \mu$. Then combining (8) and (10) gives the final estimate for $D$, namely

$$
D(\rho) \leqq K_{5}(\rho / d)^{2 \mu},
$$$$
\rho \leqq d e^{-r},
$$

and

$$
K_{5}=e\left[4 \pi \mu K^{2}+K_{3}+K_{4}\right] .
$$

We emphasize that (11) has been proved only for circles whose centers lie in $B$.

The estimate of Dirichlet growth just obtained, together with Morrey's lemma (Footnote 3 ), enables us to conclude immediately that $w$ is Hölder continuous. In fact for $z_{1}, z_{2} \in B$ we have

$$
\left|w\left(z_{1}\right)-w\left(z_{2}\right)\right| \leqq 2\left(\frac{K_{5}}{\mu}\right)^{1 / 2}\left|\frac{z_{1}-z_{2}}{d}\right|^{\mu},
$$

provided that $\left|z_{1}-z_{2}\right| \leqq d e^{-\nu}$. On the other hand, when $\left|z_{1}-z_{2}\right| \geqq d e^{-\nu}$ we have

$$
\left|w\left(z_{1}\right)-w\left(z_{2}\right)\right| \leqq 2\left|\frac{z_{1}-z_{2}}{d e^{-\nu}}\right|^{\mu}=2 e^{1 / 2}\left|\frac{z_{1}-z_{2}}{d}\right|^{\mu} .
$$

This completes the proof of Theorem 2, with $H=2 d^{-\mu}\left(K_{5} / \mu\right)^{1 / 2}$.

REMARK ON THE EXPLICIT FORM OF $H$ IN (3). If we keep track of the estimates in the above proof, we find that

$$
K_{3}+K_{4}<\pi K_{1} d^{2} /(1-\mu)^{2} .
$$

Hence

$$
\left(\frac{K_{5}}{\mu}\right)^{1 / 2}<3\left(2 K+\frac{d}{1-\mu}\left(\frac{K_{1}}{\mu}\right)^{1 / 2},\right.
$$

giving a very simple expression for $H$.

(5) Cf. M. Shiffman, Ann. of Math. vol. 48 (1947) pp. 274-284. 
2. Proof of Theorem 1. For any point set $T \subset A$ we define

$$
\omega(T)=\text { l.u.b. }\left|w\left(z_{1}\right)-w\left(z_{2}\right)\right|, \quad z_{1}, z_{2} \in T .
$$

Keeping the notation of $\S 1$, consider now circles $C_{z}$ whose centers are at least a distance $\rho$ from the boundary of $A$. We assert that

$$
\omega\left(C_{8}\right) \leqq\left(\frac{\pi D(\rho)}{2\left(1+\mu^{2}\right) \log (\rho / s)}\right)^{1 / 2}, \quad s \leqq \rho .
$$

The proof of (12) is based on a well-known argument of Lebesgue. First, since quasi-conformal mappings satisfy a maximum modulus principle $\left({ }^{6}\right)$, we have

$$
\begin{aligned}
\omega\left(C_{r}\right) & =\omega\left(\gamma_{r}\right) \leqq \frac{1}{2} \oint\left|w_{s}\right| d s, \\
& \leqq \frac{1}{2}\left[2 \pi r \oint\left|w_{8}\right|^{2} d s\right]^{1 / 2} \leqq\left[\frac{\pi r}{2\left(1+\mu^{2}\right)} \frac{d D}{d r}\right]^{1 / 2},
\end{aligned}
$$

using (4). Therefore

$$
\frac{\left[\omega\left(C_{s}\right)\right]^{2}}{r} \leqq \frac{\pi}{2\left(1+\mu^{2}\right)} \frac{d D}{d r}
$$

and (12) follows immediately by integrating both sides from $r=s$ to $r=\rho$.

The term $D(\rho)$ in (12) can be estimated by setting $K_{1}=0$ in inequality (11). Specifically, we have

$$
D(\rho) \leqq 4 \pi e \mu K^{2}\left(\rho / d^{*}\right)^{2 \mu}, \quad \rho \leqq d^{*} e^{-\nu},
$$

valid for all circles $C_{\rho}$ whose centers are at least a distance $d^{*}$ from the boundary of $A$. Now, $\left(11^{\prime}\right)$ still remains valid if the right-hand side is divided by $1+\mu^{2}$, since inequality (4) allows an extra factor $1+\mu^{2}$ to be inserted in the denominator of the right hand side of (9)-(11); thus actually

$$
D(\rho) \leqq 2 \pi e K\left(\rho / d^{*}\right)^{2 \mu},
$$$$
\rho \leqq d^{*} e^{-\nu}
$$

Now let $z_{1}$ and $z_{2}$ be two points in $B$ such that

$$
\left|z_{1}-z_{2}\right|=2 s<d e^{-2 \nu} \text {. }
$$

${ }^{(6)}$ This is well-known, but for the sake of completeness we indicate a proof. Suppose that $w$ had an interior maximum at $z=z_{0}$. Then, by an appropriate rotation of coordinates, we can assume that $u$ has a maximum at $z_{0}$. It follows that there is a level line $\gamma$ of $u$ surrounding $z_{0}$, and (compare (5)), if $C$ denotes the interior of $\gamma, \iint_{C}|\nabla w|^{2} d x d y \leqq 2 K \oint_{\gamma} u d v=$ Const. $\oint_{\gamma} d v=0$. Therefore $w \equiv$ constant in $C$. An easy argument now shows that $w \equiv$ constant throughout its domain of definition. Thus, either $w$ is a constant, or else $w$ does not take on an interior maximum. 
The midpoint of the line segment joining $z_{1}$ to $z_{2}$ is at least a distance $d^{*}$ $=.95 d$ from the boundary of $A$. Consequently, by (12) and $\left(11^{\prime \prime}\right)$,

$$
\left|w\left(z_{1}\right)-w\left(z_{2}\right)\right| \leqq \omega\left(C_{s}\right) \leqq \pi\left(\frac{e}{2 \mu \log (\rho / s)}\right)^{1 / 2}\left(\frac{\rho}{d^{*}}\right)^{\mu},
$$

provided that $s \leqq \rho \leqq d^{*} e^{-\nu}$. At this stage we are free to assign $\rho$ in any way consistent with the restriction just noted; in particular, we fix $\rho=s e^{\nu}\left(<d e^{-\nu} / 2\right)$. Then

$$
\left|w\left(z_{1}\right)-w\left(z_{2}\right)\right| \leqq \pi e\left(\frac{s}{d^{*}}\right)^{\mu}=\pi e\left|\frac{z_{1}-z_{2}}{1.9 d}\right|^{\mu} .
$$

On the other hand, when $\left|z_{1}-z_{2}\right| \geqq d e^{-2 \nu}$ we have

$$
\left|w\left(z_{1}\right)-w\left(z_{2}\right)\right| \leqq 2 e\left|\frac{z_{1}-z_{2}}{d}\right|^{\mu} \text {. }
$$

This completes the proof of Theorem 1 .

(If $A$ were convex, there would have been no need to introduce the distance $d^{*}$, but rather we could have worked always with $d$; in this case the final constant would be $2^{-\mu} \pi e$. In the conformal case, $K=1$, this gives the constant $\pi e / 2$. This is an improvement over earlier results, but still compares poorly with the "best" value 1 .)

3. Isolated singularities $\left({ }^{7}\right)$. We consider here the extension of Theorems 1 and 2 to the case where $w$ is undefined or not differentiable at isolated points of $A$, but otherwise satisfies the same hypotheses. The results of this section have been applied by Finn and Gilbarg [3] to give a very simple (rigorous) proof of the Joukowsky force formula of compressible gas dynamics, and of the uniqueness of compressible flows.

THEOREM 3. Let $w=u+i v$ satisfy the hypotheses of Theorem 1 (or Theorem 2), except at a set $T$ of isolated points in $A$. Then w can be defined, or redefined, at the points of $T$ so that the resulting function is continuous in $A$, and satisfies the conclusion of Theorem 1 (or Theorem 2).

Proof. It is sufficient to consider the case where $w$ satisfies the hypotheses of Theorem 2, the other case being exactly similar. We keep the notation of $\$ 1$.

We shall show that $D(r)$ exists and satisfies (5) for every circle $C_{r}$ whose circumference contains no points of $T$. This will prove Theorem 3 , for it follows that $D(r)$ is continuous in $r$, and that the proof of Theorem 2 applies almost unchanged. Therefore $w$ satisfies the Hölder condition (3) in $B-T$, and consequently, (by an easy argument), values of $w$ can be assigned at the points of $T$ so that the resulting function is continuous in $A$; obviously this function also satisfies (3).

(?) The developments of this section will not be needed in $\$ 4$. 
Thus, let $C_{r}$ be a circle whose circumference $\gamma_{r}$ contains no points of $T$. Suppose first that $C_{r}$ contains exactly one point $z$ of $T$ (if $C_{r}$ and $T$ are disjoint there is no problem). Let $C_{\sigma}$ be a circle of radius $\sigma$ and center $z$, and consider those values of $\sigma$ for which $C_{\sigma}$ is contained in $C_{r}$. We define $C_{\sigma r}$ $=C_{r}-C_{\sigma}$ and

$$
D(\sigma, r)=\iint_{C_{\sigma r}}|\nabla w|^{2} d x d y .
$$

Then we have (compare formula (5)),

$$
D(\sigma, r) \leqq-2 K \oint_{\gamma_{\sigma}} u d v+I,
$$

where $I$ denotes the expression on the right hand side of (5). From Schwarz's inequality and the hypothesis $|u| \leqq 1$,

$$
(D-I)^{2} \leqq-2 \pi K^{2} \sigma \frac{d D}{d \sigma},
$$

(notice that $d D / d \sigma \leqq 0$ ). Now suppose $D>I$ for some value of $\sigma$, say $\sigma=\sigma_{2}$. Then also $D>I$ for all $\sigma<\sigma_{2}$. It is therefore permissible to divide (13) by $(D-I)^{2} \sigma$ and to integrate both sides of the resulting inequality from $\sigma_{1}$ to $\sigma_{2}, \sigma_{1}<\sigma_{2}$. It follows that

$$
\log \frac{\sigma_{2}}{\sigma_{1}} \leqq \frac{2 \pi K^{2}}{D\left(\sigma_{2}, r\right)-I}
$$

But this is impossible for very small values of $\sigma_{1}$, so that by contradiction we have proved $D(\sigma, r) \leqq I$. Letting $\sigma$ tend to zero, we obtain $D(r) \leqq I$, which is the required result.

If $C_{r}$ contains more than one point of $T$, the same argument can be used to eliminate one after the other of the singular points. Since there can be at most a finite number of points of $T$ in any $C_{r}$, this completes the proof of the italicized statement and of Theorem 3.

Theorem 3 can be given a somewhat more general form if we drop the condition that $w$ be bounded. Specifically we can prove the following result.

THEOREM 4. Let $w=u+i v$ be a continuously differentiable complex function defined in the region $0<|x| \leqq 1$. Suppose that

$$
u_{x}^{2}+u_{y}^{2}+v_{x}^{2}+v_{y}^{2} \leqq 2 K\left(u_{x} v_{y}-u_{y} v_{x}\right)+K_{1}|x|^{-2 \lambda}
$$

where $K, K_{1}$, and $\lambda$ are constants, $K>1, K_{1} \geqq 0$, and $0 \leqq \lambda<1$. Also assume

$$
u=o(|z|-\mu)
$$

as $z \rightarrow 0$,

$\mu=K-\left(K^{2}-1\right)^{1 / 2}$. Then $w$ can be defined at $z=0$ so that the resulting function is continuous in $0 \leqq|z| \leqq 1$; moreover in any closed subregion of $|z|<1$, w satisfies 
a uniform Hölder condition with exponent

$$
\alpha=\operatorname{Min}(\mu, 1-\lambda),
$$

(if $1-\lambda=\mu$, the exponent must be replaced by $\mu-\epsilon$, where $\epsilon$ is an arbitrary small positive number).

Proof. We show first that if $C_{r}$ is any circle in $|z| \leqq 1$, whose circumference $\gamma_{r}$ does not contain the origin, then $D(r)$ exists and satisfies

$$
D(r) \leqq 2 K \oint_{\gamma_{r}} u d v+K_{1} \iint_{C_{r}}|z|^{-2 \lambda} d x d y .
$$

If $C_{r}$ does not contain the origin, (15) follows immediately by integrating (14). Thus suppose $C_{r}$ contains the origin. Letting $C_{\sigma}$ denote a circle of radius $\sigma$ and center $z=0$, we have, as in the proof of Theorem 3 ,

$$
D(\sigma, r) \leqq-2 K \oint_{\gamma_{\sigma}} u d v+J
$$

where $J$ denotes the expression on the right side of (15). Now by hypothesis

$$
u^{2} \leqq \epsilon(|z|)|z|^{-2 \mu}
$$

where $\epsilon(|z|) \rightarrow 0$ as $z \rightarrow 0$; without loss of generality we may assume that $\epsilon(|z|)$ is monotonically increasing. It follows from (16) and (17) that

$$
(D-J)^{2} \leqq-8 \pi K^{2} \epsilon(\sigma) \sigma^{1-2 \mu} \frac{d D}{d \sigma} \text {. }
$$

Now suppose for contradiction that $D>J$ for some value of $\sigma$, say $\sigma=\sigma_{0}$. Then also $D>J$ for all $\sigma<\sigma_{0}$, and we have

$$
\sigma^{2 \mu-1} \leqq-8 \pi K^{2} \epsilon(\sigma) \frac{1}{(D-J)^{2}} \frac{d D}{d \sigma}, \quad \sigma<\sigma_{0},
$$

Let us integrate this from $\sigma_{1}$ to $\sigma, \sigma_{1}<\sigma<\sigma_{0}$, holding the argument of $\epsilon$ fixed at the upper limit of integration. We arrive at

$$
\sigma^{2 \mu}-\sigma_{1}^{2 \mu} \leqq 16 \pi K^{2} \mu \epsilon(\sigma)(D-J)^{-1}
$$

and therefore, letting $\sigma_{1} \rightarrow 0$,

$$
D-J \leqq 16 \pi K^{2} \mu \epsilon(\sigma) \sigma^{-2 \mu}, \quad \sigma<\sigma_{0} .
$$

On the other hand, in the same way that inequality (5) implied (7), inequality (16) implies

$$
D-J \leqq-\frac{\sigma}{2 \mu} \frac{? d D}{d \sigma}+\frac{2 \pi K_{1 \mu}}{1:-\mu^{2}} \sigma^{2-2 \lambda} .
$$


A simple integration leads to

$$
D-J \geqq\left\{D\left(\sigma^{\prime}, r\right)-J-\text { Const. } \sigma^{2-2 \lambda}\right\}\left(\sigma^{\prime} / \sigma\right)^{2 \mu}, \quad \sigma<\sigma^{\prime} .
$$

Now let $\sigma^{\prime}$ be fixed so small that the expression in braces is positive. Then

$$
D-J \geqq \text { Const. } \sigma^{-2 \mu}, \quad \text { Const. }>0, \quad \sigma<\sigma^{\prime} .
$$

But this contradicts (18) for sufficiently small $\sigma$ : hence $D(\sigma, r) \leqq J$. Letting $\sigma \rightarrow 0$ gives, finally, $D(r) \leqq J$, i.e. equality (15).

We can now proceed as in the proof of Theorem 2. Let $B$ be an arbitrary closed subregion of $|z|<1$, and let $d$ be the distance from $B$ to $|z|=1$. For any circle $C_{r}$ with center in $B$ we find, as in Theorem 2,

$$
D(r) \leqq \frac{r}{2 \mu} \frac{d D}{d r}+\frac{K_{1} \mu r}{1-\mu^{2}} \oint_{\gamma_{r}}|z|^{-2 \lambda} d s+K_{1} \iint_{C_{r}}|z|^{-2 \lambda} d x d y,
$$

provided that $\gamma_{r}$ does not pass through the origin. Setting

$$
E(r)=\iint_{C_{r}}|z|^{-2 \lambda} d x d y,
$$

(19) can be written in the form

$$
-\frac{d}{d r}\left(r^{-2 \mu} D\right) \leqq K_{6} \frac{d}{d r}\left(r^{-2 \mu} E\right)+K_{7} r^{-2 \mu-1} E,
$$

where $K_{6}$ and $K_{7}$ are constants depending only on $K$ and $K_{1}$. Integrating both sides from $\rho$ to $d$, we get

$$
\rho^{-2 \mu} D(\rho)-d^{-2 \mu} D(d) \leqq K_{6} d^{-2 \mu} E(d)+K_{7} \int_{\rho}^{d}{ }^{-2 \mu-1} E d r .
$$

Now it is easy to see that $E(r)$, for fixed $r$, is greatest when the center of $C_{r}$ is at the origin. Consequently

$$
\int_{\rho}^{d} r^{-2 \mu-1} E d r \leqq \frac{\pi}{2(1-\lambda)|1-\lambda-\mu|} \begin{cases}d^{2-2 \lambda-2 \mu} & \text { if } \mu<1-\lambda, \\ \rho^{2-2 \lambda-2 \mu} & \text { if } \mu>1-\lambda,\end{cases}
$$

provided that $1-\lambda \neq \mu$. Moreover, $D(d)$ is uniformly bounded by $\iint_{|z| \leq 1}|\nabla w|^{2} d x d y$. Therefore

$$
D(\rho) \leqq \text { Const. } \rho^{2 \alpha}, \quad \rho \leqq d, 1-\lambda \neq \mu
$$

$(\alpha=\operatorname{Min}(\mu, 1-\lambda))$. We conclude from Morrey's lemma that $w$ is uniformly Hölder continuous in $B$ with exponent $\alpha$. The exceptional case $1-\lambda=\mu$ is handled by replacing $\lambda$ in (14) by $\lambda+\epsilon$, and Theorem 4 is completely proved.

4. One-to-one quasi-conformal mappings. In this section we consider oneto-one quasi-conformal mappings of the open unit disk onto itself. The ap- 
parently more general situation described in the introduction can be reduced to the present case by means of conformal mapping techniques.

THEOREM 5. Let $w(z)$ be a one-to-one quasi-conformal mapping of $|z|<1$ onto $|w|<1$, such that $w(0)=0$. Then $w$ can be extended to a one-to-one continuous mapping of $|z| \leqq 1$ onto $|w| \leqq 1$, satisfying the Hölder condition

$$
\left|w\left(z_{1}\right)-w\left(z_{2}\right)\right| \leqq H\left|z_{1}-z_{2}\right|^{\mu},
$$

where $\mu=K-\left(K^{2}-1\right)^{1 / 2}$, and $H$ is an absolute constant $\left({ }^{8}\right)$.

Proof. We note first of all that the Dirichlet integral is bounded:

$$
\iint_{|s| \leq 1}|\nabla w|^{2} d x d y \leqq 2 K \iint_{|z| \leq 1}\left(u_{x} v_{y}-u_{x} v_{y}\right) d x d y=2 K \pi,
$$

using the fact that the mapping is one-to-one.

Now let $z_{0}$ be an arbitrary point on $|z|=1$, let $C_{r}^{\prime}$ denote the part of the circle $\left|z-z_{0}\right| \leqq r$ which lies in $|z| \leqq 1$, and let $\gamma_{r}^{\prime}$ denote the part of the boundary of $C_{r}^{\prime}$ in $|z|<1$. Then for $r<1$ we have

$$
\omega\left(C_{r}^{\prime}\right) \leqq l_{r},
$$

where $l_{r}$ is the length of the image of $\gamma_{r}^{\prime}$. To prove (21), suppose first that $l_{r}<2$. Then the image of $\gamma_{r}^{\prime}$ cannot intersect a diameter of $|w| \leqq 1$ on both sides of the origin, and it easily follows that

$$
\omega\left(C_{r}^{\prime}\right)=\omega\left(\gamma_{r}^{\prime}\right) \leqq l_{r} .
$$

On the other hand, if $l_{r} \geqq 2$, then $\omega\left(C_{r}^{\prime}\right) \leqq 2 \leqq l_{r}$, and (21) is verified in all cases. Next, we assert that

$$
\omega\left(C_{r}^{\prime}\right) \leqq \pi\left(\frac{1}{\mu \log (1 / r)}\right)^{1 / 2}
$$

The proof is very similar to the proof of (12): we have

$$
\omega\left(C_{r}^{\prime}\right) \leqq l_{r}=\int_{\theta_{1}(r)}^{\theta_{2}(r)}\left|w_{s}\right| d s \leqq\left[\frac{\pi r}{1+\mu^{2}} \frac{d D^{\prime}}{d r}\right]^{1 / 2} ;
$$

integrating and using (20) leads at once to (22).

From (22) it, follows that any sequence of points $z_{n} \rightarrow z_{0}$ has as image a

(8) Lavrentieff has proved a similar result, but with $H$ depending on $K$, and with a smaller exponent. Ahlfors improved Lavrentieff's result to the extent of getting the best exponent, although his coefficient still depends on $K$. After work on this paper was completed, it was found that proofs of this theorem had been given by Yûjôbô and by Mori, and that Mori had proved $H \leqq 16$, a best possible result. Our coefficient is considerably larger than this best value, but our proof is relatively so simple that its inclusion still seems justified. 
Cauchy sequence of points $w_{n}$. Therefore the points $w_{n}$ tend to a limit point $w_{0}$, which is obviously on $|w|=1$ and uniquely determined by $z_{0}$. Since $z_{0}$ was any point on $|z|=1$, and since the inverse mapping $w \rightarrow z$ has the same properties as the mapping $z \rightarrow w$, this proves the first part of Theorem 5 .

We turn now to the Hölder continuity of the mapping $|z| \leqq 1$ onto $|w| \leqq 1$. Because this mapping is one-to-one and takes the origin into itself, it can be extended by the relation $w(1 / \bar{z})=(1 / \bar{w}(z))$ to a one-to-one continuous mapping of $|z|<\infty$ onto $|w|<\infty$. This new mapping, which we also call $w(z)$, is quasi-conformal with constant $K$, everywhere except on $|z|=1$. Let us set $d=\exp \left(\neg \pi^{2} N^{2} / \mu\right)$, where $N>1$ is a numerical constant to be assigned later. Then in the disk $|z| \leqq 1+d$, we have

$$
|w|<\left[\min _{|z|=1-d}|w|\right]^{-1} \leqq\left[1-\pi\left(\frac{1}{\mu \log (1 / d)}\right)^{1 / 2}\right]^{-1}=\frac{N}{N-1},
$$

by (22).

At this stage, in spite of the fact that $w(z)$ may not be differentiable on $|z|=1$, the formal operations of $\S \S 1$ and 2 are nevertheless applicable (see \$1 of the Appendix). In particular, we may apply Theorem 1 with $A$ as the disk $|z| \leqq 1+d$. It follows that, for any two points $z_{1}$ and $z_{2}$ in $|z| \leqq 1$,

$$
\left|w\left(z_{1}\right)-w\left(z_{2}\right)\right|<\pi e \frac{N}{N-1}\left|\frac{z_{1}-z_{2}}{d}\right|^{\mu}=\pi e \frac{N}{N-1} e^{\pi^{2} N^{2}}\left|z_{1}-z_{2}\right|^{\mu} .
$$

Setting $N=1+1 / 2 \pi^{2}$ completes the proof of Theorem 5 .

\section{APPENDIX}

A1. We wish to prove that the inequalities (8) and (10) on growth of the Dirichlet integral of $w$ remain valid under the weakened differentiability requirements (i)-(iii) of the introductory section of the paper. For simplicity we shall prove (10) under the assumption $K_{1}=0$, the changes necessary for the other cases being obvious.

Let us approximate $w$ in the square integral norm of its derivatives by continuously differentiable functions $w^{(h)}$. Thus, $\iint_{A}\left|\nabla\left(w-w^{(h)}\right)\right|^{2} d x d y \rightarrow 0$, $\sup _{A}\left|w-w^{(h)}\right| \rightarrow 0$ as $h \rightarrow 0$. For the functions $w^{(h)}=u^{(h)}+i v^{(h)}$ we have the analogue of (5),

$$
\begin{aligned}
Q^{(h)}(r) & =\iint_{c_{r}}\left(u_{x}^{(h)} v_{y}^{(h)}-u_{y}^{(h)} v_{x}^{(h)}\right) d x d y=\frac{1}{2} \oint\left[u^{(h)} d v^{(h)}-v^{(h)} d u^{(h)}\right] \\
& \leqq \frac{1}{2}\left[\oint\left|w^{(h)}\right|^{2} d s\right]^{1 / 2}\left[\oint\left|w_{\varepsilon}^{(h)}\right|^{2} d s\right]^{1 / 2} \cdot
\end{aligned}
$$

Since $|w|<1$ in $A$, 


$$
Q^{(k)}(r) \leqq \frac{1+\epsilon}{2}[2 \pi r]^{1 / 2}\left[\frac{d D^{(h)}}{d r}\right]^{1 / 2}
$$

where $\epsilon \rightarrow 0$ as $h \rightarrow 0$ and

$$
D^{(h)}(r)=\iint_{C_{r}}\left|\nabla w^{(h)}\right|^{2} d x d y .
$$

We now square both sides of (A2) and integrate between the limits $r$ and $r+\lambda, \lambda$ fixed( $\left(^{5}\right)$ :

$$
\int_{r}^{r+\lambda}\left[Q^{(h)}(\rho)\right]^{2} d \rho \leqq\left(\frac{1+\epsilon}{2}-\right)^{2} \cdot 2 \pi(r+\lambda)\left[D^{(h)}(r+\lambda)-D^{(h)}(r)\right] .
$$

We are now at liberty to let $h \rightarrow 0$. Thus

$$
\int_{r}^{r+\lambda}[Q(\rho)]^{2} d \rho \leqq \frac{\pi}{2}(r+\lambda)[D(r+\lambda)-D(r)]
$$

where $Q(\rho)=\iint_{c_{\rho}}\left(u_{x} v_{y}-u_{y} v_{x}\right) d x d y$. Using (2) we find

$$
\int_{r}^{r+\lambda}[D(\rho)]^{2} d \rho \leqq 2 K^{2} \pi(r+\lambda)[D(r+\lambda)-D(r)] .
$$

Set $F(r)=\int_{r}^{r+\lambda} D(\rho) d(\rho)$. Using Schwarz's inequality,

$$
[F(r)]^{2} \leqq 2 K^{2} \pi \lambda(r+\lambda) F^{\prime}(r) .
$$

Integrating,

$$
\frac{1}{F(r)}-\frac{1}{F(R)} \geqq \frac{1}{2 K^{2} \pi \lambda} \log \frac{R+\lambda}{r+\lambda}, \quad R>r
$$

which implies

$$
\frac{1}{\lambda D(r)} \geqq \frac{1}{2 K^{2} \pi \lambda} \log \frac{R+\lambda}{r+\lambda} .
$$

Letting $\lambda \rightarrow 0$, we obtain (10) for the case considered.

A2. Because Morrey's lemma is so important in the paper, we state and prove it here $\left({ }^{\circ}\right)$.

LEMMA (MORREY). Let $w(z)$ be a continuous differentiable complex function defined in a domain $A$ of the z-plane. Let $B$ be a closed subregion of $A$, and $d$ the distance from $B$ to the boundary of $A$. Suppose that there are positive constants $L, \mu$, and $r_{0}\left(r_{0} \leqq d\right)$, such that

(P) The method proof is due to Shiffman $[8$, p. 651]. 


$$
D(r)=\iint_{C_{r}}|\nabla w|^{2} d x d y \leqq L r^{2 \mu}
$$

for all circles $C_{r}$ with center in $B$ and radius $r \leqq r_{0}$. Then w satisfies the following $H$ ölder condition in $B$,

$$
\left|w\left(z_{1}\right)-w\left(z_{2}\right)\right| \leqq 2\left(\frac{L}{\mu}\right)^{1 / 2}\left|z_{1}-z_{2}\right|^{\mu}, \quad\left|z_{1}-z_{2}\right| \leqq r_{0} .
$$

Proof. Denote the points $z_{1}, z_{2}$ by $P$ and $Q$, respectively, and let $L$ be the perpendicular bisector of the line segment $P Q$. Select a point $S$ on $L$ such that $\bar{P} \bar{S}=\bar{Q} \bar{S} \leqq \bar{P} \bar{Q} \leqq r_{0}$. Then we have

$$
w(P)-w(Q)=\int_{P S} w_{r} d r-\int_{Q S} w_{r} d r
$$

where the integrations are taken along the line segments $P S$ and $Q S$, respectively, and $r$ represents the distance from $P$ and $Q$, respectively. We denote the angle $Q P S$ by $\theta$, so that (A4) holds for $-\pi / 3 \leqq \theta \leqq \pi / 3$. Taking absolute values in (A4), and integrating with respect to $\theta$ from $-\pi / 3$ to $\pi / 3$, gives

$$
|w(P)-w(Q)| \leqq \frac{3}{2 \pi} \iint|\nabla w| d r d \theta+\frac{3}{2 \pi} \iint|\nabla w| d r d \theta,
$$

where the integrals may be taken over the values $0 \leqq r \leqq \bar{P} \bar{Q}$ and $-\pi / 3 \leqq \theta$ $\leqq \pi / 3$. By the Schwarz inequality

$$
\left(\iint|\nabla w| d r d \theta\right)^{2} \leqq \iint r^{\mu-1} d r d \theta \cdot \iint|\nabla w|{ }^{2} r^{1-\mu} d r d \theta .
$$

Now

$$
\iint r^{\mu-1} d r d \theta=\frac{2}{3} \pi \cdot \frac{1}{\mu} \cdot \overline{P Q}^{\mu}
$$

and by an integration by parts,

$$
\iint|\nabla w|{ }^{2} r^{1-\mu} d r d \theta \leqq D(\overline{P Q}) \cdot \overline{P Q} \bar{Q}^{-\mu}+\mu \int_{0}^{\overline{P Q}} D(r) \cdot r^{-\mu-1} d r \leqq 2 L \cdot \overline{P Q^{\mu}} .
$$

Inserting these estimates in (A5), we obtain

$$
|w(P)-w(Q)| \leqq\left(\frac{12}{\pi}\right)^{1 / 2}\left(\frac{L}{\mu}\right)^{1 / 2} \overline{P Q}^{\mu} .
$$

REMARK. If $w$ is not continuously differentiable, but instead satisfies conditions (i)-(iii) of the introductory section, Morrey's lemma continues to hold. The proof is (essentially) the same as above. 
Note ADDED IN PROOF. Independently of our work, Philip Hartman has obtained a result quite similar to our Theorem 2 (cf. Duke Math. J. vol. 25 (1957) pp. 57-66).

\section{REFERENCES}

1. L. Ahlfors, On quasi-conformal mappings, Journal d'Analyse Mathématique vol. 3 (1953-1954) pp. 1-58.

2. R. Caccioppoli, Fondamenti per una teoria generale delle funzioni psuedoanalitiche di una variabile complessa, Atti della Accademia Nazionale dei Linsei, Rendiconti, de Scienze, Fisiche, Matematiche e Naturali vol. 13 (1952) pp. 197-204.

3. R. Finn and D. Gilbarg, Uniqueness and the force formulas for plane subsonic flows, Trans. Amer. Math. Soc. vol. 88 (1958) pp. 375-379.

4. Hardy, Littlewood, and Polya, Inequalities, Cambridge, 1934.

5. M. Lavrentieff, A fundamental theorem of the theory of quasi-conformal mappings of twodimensional regions, Izvestiya Akademii Nauk SSSR vol. 12 (1948) pp. 513-544, (Amer. Math. Soc. Translations, no. 29).

6. C. B. Morrey, Jr., On the solutions of quasilinear elliptic partial differential equations, Trans. Amer. Math. Soc. vol. 43 (1938) pp. 126-166.

7. L. Nirenberg, On nonlinear elliptic partial differential equations and Hölder continuity, Communications on Pure and Applied Mathematics vol. 6 (1953) pp. 103-156.

8. M. Shiffman, On the existence of subsonic flows of a compressible fluid, Journal of Rational Mechanics and Analysis vol. 1 (1952) pp. 605-652.

9. J. Hersch and A. Pfluger, Généralisation du lemme de Schwarz et du principe de la mesure harmonique pour les fonctions pseudo-analytiques, C. R. Acad. Sci. Paris vol. 234 (1952) pp. 4345.

10. J. Hersch, Contribution d la theorie des fonctions pseudo-analytiques, Comment. Math. Helv. vol. 30 (1956) pp. 1-19.

11. A. Mori, On an absolute constant in the theory of quasi-conformal mappings, Journal Mathematical Society of Japan vol. 8 (1956) pp. 156-166.

12. Z. Yajổbô, On pseudo-regular functions, Comment. Math. Univ. St. Paul. vol. 1 (1953) pp. $67-80$.

California Institute of Technology, Pasadena, Calif.

UNIVERSITY OF MiNNESOTA, MinNeAPOLIS, Minn. 